\title{
Drop metastasis from hepatocellular carcinoma after percutaneous radiofrequency ablation therapy
}

Sang Kuon Lee ${ }^{1}$ and Myeong Jun Song ${ }^{2}$

Departments of ${ }^{1}$ Surgery and ${ }^{2}$ Internal Medicine, College of Medicine, Daejeon St. Mary's Hospital, The Catholic University of Korea, Daejeon, Korea

Received: November 23, 2015 Revised : January 6, 2016 Accepted: January 13, 2016

\section{Correspondence to} Myeong Jun Song, M.D.

Tel: +82-42-220-9114

Fax: +82-42-220-9565

E-mail:mjsong95@gmail.com
For treating hepatocellular carcinoma (HCC), radiofrequency ablation (RFA), a nonsurgical locoregional therapy, is frequently chosen as the first treatment modality because it is effective and less invasive, with preservation of the remnant hepatic parenchyma. Tumor implantation along needle tract or on other sites is a rare complication of RFA. We present a patient with HCC who developed a drop metastasis into the peritoneal cavity.

A 62-year-old male cirrhotic patient with hepatitis B was diagnosed with HCC. On the computed tomography
(CT) scan, a 2-cm solitary tumor was found in hepatic segment VII, compatible with HCC (Fig. 1A). Laboratory findings showed an elevated protein induced by vitamin $\mathrm{K}$ absence/antagonist-II (PIVKA-II) level (586 mAU/mL), with a normal $\alpha$-fetoprotein level. The patient was treated with percutaneous RFA. Artificial ascites was created for better visualization of the tumor. The treatment was successful, with complete necrosis of the tumor (Fig. 1B). Twelve months later, a 6-cm lobulated heterogeneous highly enhancing mass was found on the anterior aspect of the

Figure 1. Computed tomography(CT) scans show a 2-cm hepatic tumor (arrows) in segment VII, (A) pre- and (B) post-radiofrequency ablation views. One year later, (C) axial and (D) coronal CT scans show a 6-cm lobulated heterogeneous highly enhancing mass (arrows) on anterior aspect of ascending colon.
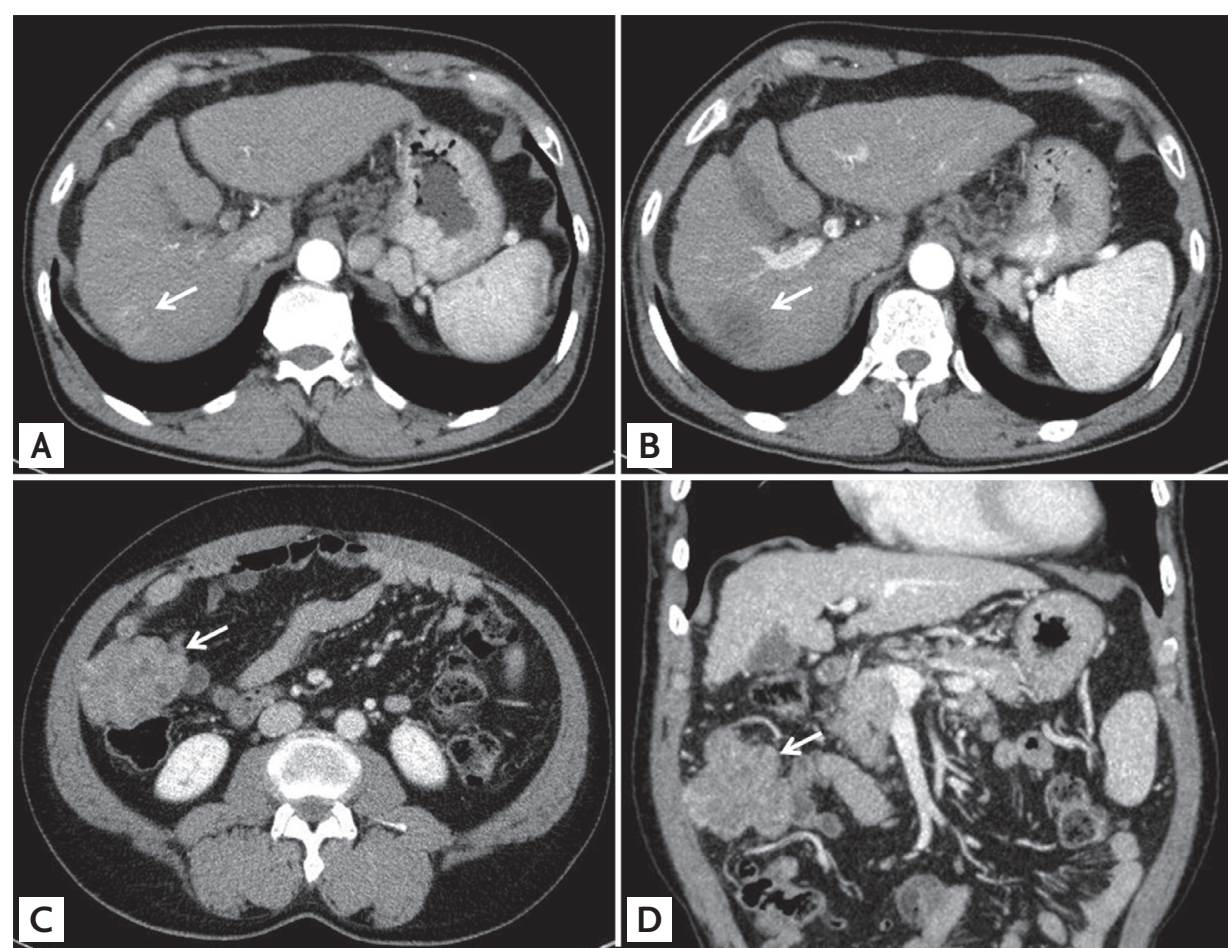

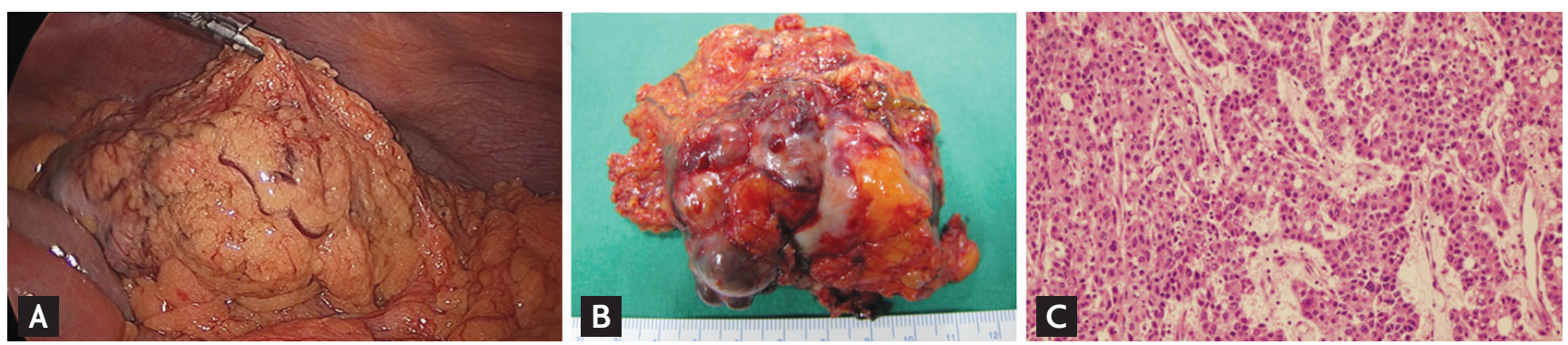

Figure 2. (A) A 6-cm ovoid reddish tumor completely wrapped in the omentum is seen in the right paracolic gutter. (B) Pathologic specimen shows a solid and necrotic mass with histological diagnosis of metastatic hepatocellular carcinoma. (C) Histological finding of hepatocellular carcinoma with trabecular pattern $(\mathrm{H} \& \mathrm{E}, \times 200)$.

ascending colon on a routine follow-up CT scan (Fig. $1 \mathrm{C}$ and $1 \mathrm{D}$ ). Colonofiberscopy showed no luminal mass or extrinsic compression sign.

Laparoscopic exploration was performed and a $6 \times$ 4-cm ovoid highly vascular tumor completely wrapped in the omentum was found in the right paracolic gutter (Fig. 2A and 2B). The tumor showed no colonic invasion, was easily dissected free from the surrounding tissues, and was completely removed. Pathologically, it was diagnosed as metastatic HCC (Fig. 2C). Four months after surgery, there is no evidence of tumor recurrence on CT scan, and the PIVKA-II level has normalized.

Drop metastasis after RFA may occur when handling the needle. Cancerous cells may fall and seed the peritoneal cavity forming metastatic deposits. Therefore, to prevent cancer spillage, it is important to minimize the use of artificial ascites and avoid needle entry through the free peritoneal cavity.

\section{Conflict of interest}

No potential conflict of interest relevant to this article was reported. 